\title{
Commensurate Composite Fermions in Weak Periodic Electrostatic Potentials: Direct Evidence of a Periodic Effective Magnetic Field
}

\author{
J. H. Smet, ${ }^{1}$ S. Jobst, ${ }^{2}$ K. von Klitzing, ${ }^{1}$ D. Weiss, ${ }^{2}$ W. Wegscheider ${ }^{3}$ and V. Umansky ${ }^{4}$ \\ ${ }^{1}$ Max-Planck-Institut für Festkörperforschung, Heisenbergstraße 1, D-70569 Stuttgart, Germany \\ ${ }^{2}$ Institut für Experimentelle und Angewandte Physik, Universität Regensburg, D-93040 Regensburg, Germany \\ ${ }^{3}$ Walter-Schottky-Institut, Technische Universität München, Am Coulombwall, D-85748 Garching, Germany \\ ${ }^{4}$ Braun Center for Submicron Research, Weizmann Institute of Science, Rehovot 76100, Israel
}

(Received 14 January 1999)

\begin{abstract}
Within the composite fermion model, it is anticipated that a periodic variation of the density in a two-dimensional electron system at half filling produces a periodic fictitious magnetic field. In regions of smaller density fictitious flux quanta are missing to compensate the external magnetic field. The observation of magnetic commensurability minima in the dc-transport response furnishes evidence for this fundamental aspect of the composite fermion picture. In general, composite fermions in these modulated systems behave largely equivalent to electrons moving in a periodic magnetic field.
\end{abstract}

PACS numbers: 73.40.Hm, 71.10.Pm, 73.50.Jt

Substantial experimental support has been gathered in recent years that the gapless state of a strongly interacting two-dimensional electron system (2DES) at the Landau filling factor $\nu=1 / 2$ behaves largely equivalent to a metal at zero magnetic field. The metallic state is described in terms of composite fermions (CFs), quasiparticles assembled from one electron and two fictitious flux quanta, pointing in the direction opposite to the external applied magnetic field [1]. Away from half filling, composite fermions move in a reduced, so-called effective, magnetic field $B_{\text {eff }}$. The latter is determined both by the external magnetic field $B$ and by the density $n_{s}$,

$$
B_{\text {eff }}=B-B_{1 / 2}=B-2 n_{s} \Phi_{0},
$$

where $B_{1 / 2}$ is the external field required to reach half filling and $\Phi_{0}=h / e$ is the unit of flux. This model has proved highly successful in accounting for results obtained in antidot lattices, surface acoustic wave experiments, and transverse magnetic focusing geometries around $\nu=1 / 2$, to name but a few [2].

According to Eq. (1), in regions with a deficit or surplus $\Delta n_{s}$ of composite fermions, with respect to the average density $\bar{n}_{s}$, a number of fictitious flux quanta $2 \Delta n_{s} \Phi_{0}$ are either missing or superfluous to compensate the external magnetic field at half filling. They produce a strong local effective magnetic field $\Delta B_{\text {eff }}=B_{1 / 2} \Delta n_{s} / \bar{n}_{s}$. Very recently, experiments have been devised to test this fundamental property inherent to the flux attachment in the composite fermion picture. To this end, the influence of a periodic variation of the density on the dc-transport properties and on the propagation of surface acoustic waves (SAW) across the modulated two-dimensional system has been studied. It was anticipated that the periodic effective magnetic field induced by the density modulation would give rise to magnetic commensurability effects in $\mathrm{dc}$ transport [3-5]. However, their observation remained elusive. The dc-transport experiments performed on samples with metallic gates yielded no new features and no large systematic effects [6], whereas samples that underwent a shallow reactive ion etch displayed consistently a strong positive magnetoresistance emanating from half filling [7]. The SAW experiments showed empirically features at $\nu=1 / 2$ that resemble those observed at fractional quantum Hall states [6]. In this work, by systematically varying both the amplitude $\Delta n_{s}$ and the period of the periodic density perturbation, we succeeded in uncovering magnetic commensurability effects, a clear signature of the flux attachment. These modulated systems near $\nu=1 / 2$ have been addressed theoretically by Mirlin et al. [8] and von Oppen et al. [9] using a Boltzmann equation approach within the composite fermion framework. For sufficiently small values of $\Delta n_{s}$, we find satisfactory agreement between the experimental traces and analytical expressions derived in Ref. [8].

The periodic density modulation has been implemented in two ways on GaAs/AlGaAs heterostructures with electron densities between $(1.4-2.2) \times 10^{11} \mathrm{~cm}^{-2}$ and electron mobilities of $(5.2-11.3) \times 10^{6} \mathrm{~cm}^{2} / \mathrm{V} \mathrm{s}$ prior to processing. In one method, an array of lines written by standard electron beam lithography is transferred onto the heterostructure with the aid of a shallow reactive ion etch. The difference in Fermi level pinning and surface condition between the GaAs cap layer and Al$\mathrm{GaAs}$ produces a periodic change in the conduction band profile. The amplitude of the density modulation can be tuned to some extent by varying the etch depth. A less invasive technique uses holographic illumination inside a ${ }^{3} \mathrm{He}$ cryostat (see inset of Fig. 1) [10]. It exploits the persistent photoionization of $D X$ centers in AlGaAs-based heterostructures after brief illumination at low temperatures. Two beams of a coherent light source (He-Ne laser) generate an interference pattern with a period depending on the wavelength and the angle of incidence of the two beams and induce a persistent periodic variation in the concentration of the ionized $D X$ centers, thereby imposing 


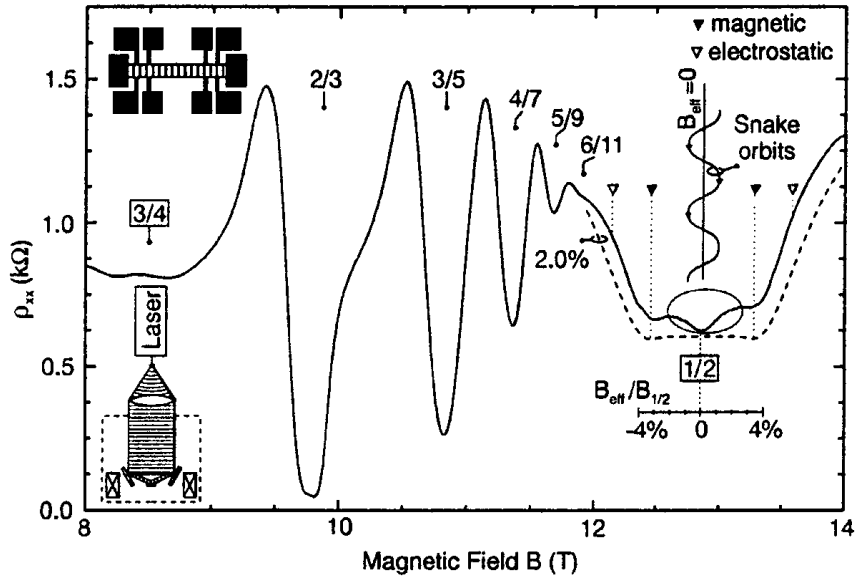

FIG. 1. The longitudinal resistance $\rho_{x x}$ near half filling for a periodic modulation of the density (top left inset) imposed by holographic illumination (bottom left inset) with a period $a=337 \mathrm{~nm}$. The response is composed of two contributions: a positive magnetoresistance attributed to CFs moving along snake orbits (shaded area) and a dramatic increase in $\rho_{x x}$ when the $\mathrm{CF}$ cyclotron diameter drops below $5 / 4 \times a$ associated with an additional drift of the centers of motion of the CFs due to the periodic effective magnetic field. The dashed curve is a fit to the experimental data for $\eta=2.0 \%$ using the analytical expressions derived in Ref. 8.

a weak periodic potential in the 2DES. If saturation is avoided, holographic illumination ensures a sinusoidal potential modulation. The periodicities investigated cover the range from $490 \mathrm{~nm}$ down to $280 \mathrm{~nm}$. For the studied heterostructures, periods, and etch depths, both techniques give similar results. Unless otherwise stated, it will hereafter be assumed that the current flows perpendicular to the direction of the stripes of the one-dimensional gratings.

The longitudinal resistance of a 2DES subjected to both a weak one-dimensional periodic potential or a periodic magnetic field and a tunable homogeneous perpendicular magnetic field $B$ displays $1 / B$-periodic oscillations which reflect the commensurability between the classical cyclotron radius $R_{\mathrm{c}}$ at the Fermi energy $E_{\mathrm{F}}$ and the period $a$. Minima appear in the resistance when

$$
2 R_{\mathrm{c}}=a(\lambda \mp 1 / 4)
$$

holds, where $\lambda=1,2, \ldots$ is an integer oscillation index, and with the "-" or "+" sign for a periodic electrostatic and magnetic field, respectively [11]. Classically, the additional contribution to the resistivity is attributed to a drift of the centers of motion of the electrons along the direction of the stripes induced by the crossed periodic electric field and external magnetic field or the periodic variation of the Lorentz force. However, when Eq. (2) is satisfied, the electron trajectories average away the effect of the periodic fields, and the resistivity drops back to its zero field value. The position of the commensurability minima on the magnetic field abscissa allows one to dis- tinguish between electrostatic and magnetic modulation. An example of low field data for a 2DES subjected to a periodic electrostatic potential is depicted in the inset of Fig. 3(a) (below).

A similar scenario should hold for composite fermions around $\nu=1 / 2$. The commensurability conditions of Eq. (2) remain valid if the CF cyclotron radius is substituted for the electron cyclotron radius $R_{\mathrm{c}}$. Even though in this work we impose only a periodic change in the conduction band profile (in other words, the density) with an amplitude $\Delta V=E_{\mathrm{F}} \Delta n_{s} / \bar{n}_{s}$, it is expected that a periodic effective magnetic field is simultaneously generated for CFs with a correlated amplitude $\Delta B_{\text {eff }}=B_{1 / 2} \Delta n_{s} / \bar{n}_{s}$. The ratio $\Delta n_{s} / \bar{n}_{s}$ will hereafter be referred to as $\eta$. We assert that the response to this periodic perturbation of the 2DES near half filling is dominated by the periodic effective magnetic field and displays the primary magnetic commensurability minima $(\lambda=1)$ [12]. Figure 1 shows the longitudinal resistance for a periodic density variation with a period $a$ of $337 \mathrm{~nm}$ obtained with the aid of holographic illumination. Solid and open triangles denote the expected positions of the magnetic and electric commensurability minima with index $\lambda=1$, respectively. To further

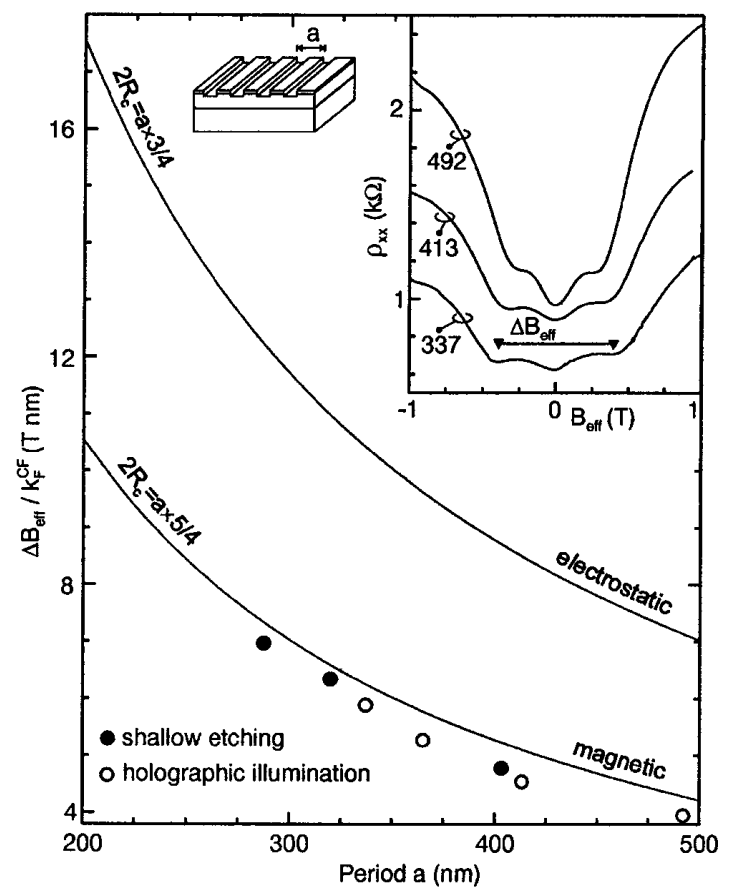

FIG. 2. The magnetic field difference between the primary minima in $\rho_{x x}$ due to commensurate orbits as a function of the modulation period $a$ for etched gratings and holographic illumination. The ordinate was divided by the CF Fermi wave vector, assuming complete spin polarization, for the sake of comparing samples with different electron density. The theoretical dependencies for a periodic electrostatic potential or a periodic magnetic field are also included (solid curves). The inset displays the shift of the minima with decreasing period (indicated in $\mathrm{nm}$ ) for some of the data with holographic modulation. 


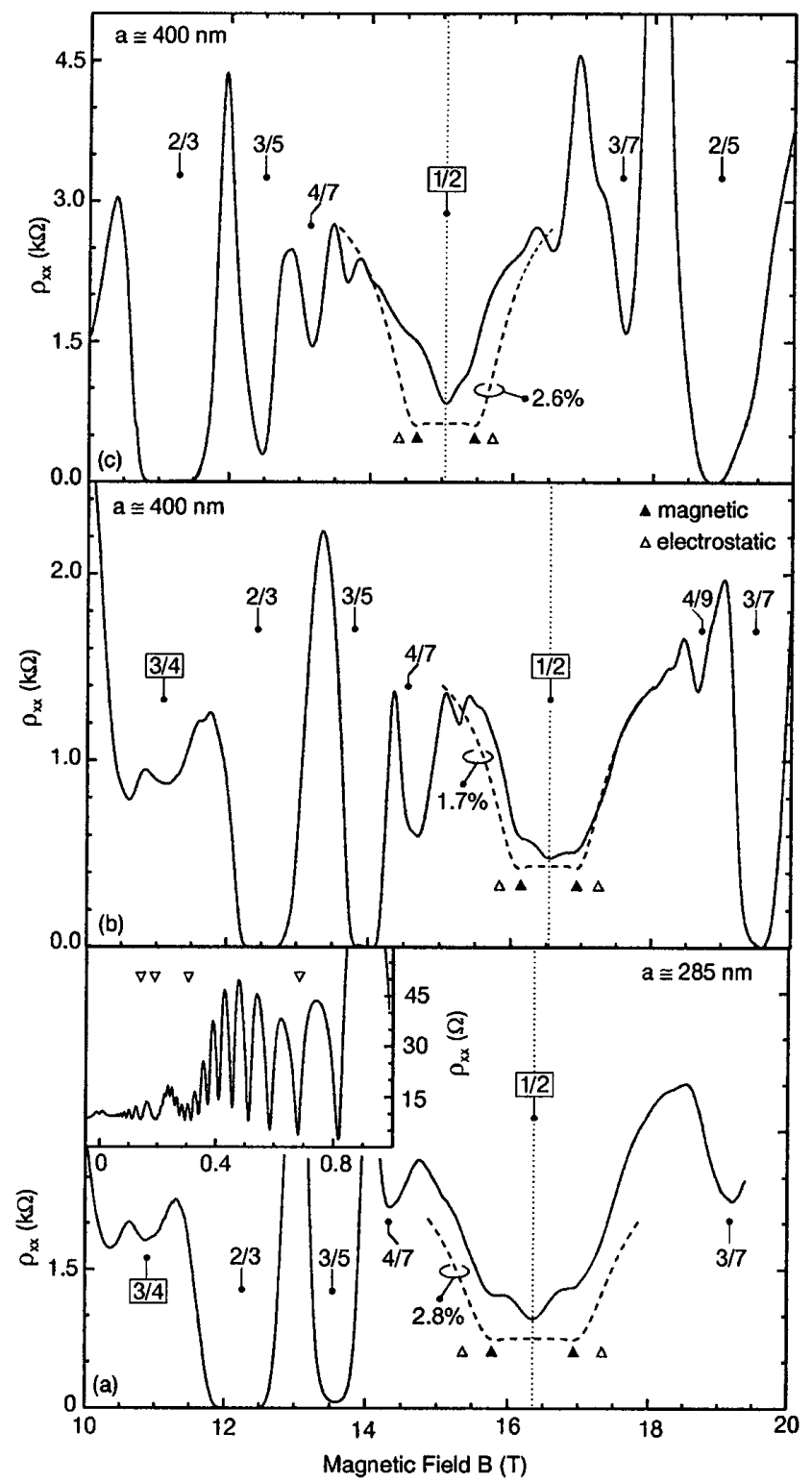

FIG. 3. The longitudinal resistance $\rho_{x x}$ for a set of etched gratings with periods of $285 \mathrm{~nm}$ (a) and $400 \mathrm{~nm}$ [(b),(c)]. The etch depth for samples (b) and (c) is different. The inset in graph (a) shows the low field data. There are no signs for higher harmonics in the electrostatic potential. The dashed curves are best fits to the data. The modulation amplitude $\eta$ is given in $\%$.

support our assertion, the same experiment was repeated for other periods (examples for holographic modulation are shown in the inset to Fig. 2 and for etched gratings in Fig. 3). The result is summarized in Fig. 2. In all cases, the observed minima lie at $2 R_{\mathrm{c}}=(1+1 / 4) a$ and make a strong case for magnetic commensurability effects.

Mirlin and co-workers described this system theoretically within the $\mathrm{CF}$ framework in terms of fermions subjected to a modulated magnetic field and scattered by a random magnetic field using the Boltzmann equation approach [8]. They derived approximate analytical expres- sions that lend themselves for fitting to the experimental data. Their main conclusions are that in the dc-transport case the influence of the periodic electrostatic potential can be ignored [13] and that the contribution to the resistivity induced by the periodic magnetic field with wave vector $\vec{p}$ can approximately be written as [8]

$$
\Delta \rho_{x x} \approx \frac{\eta^{2}}{2}\left(\frac{2 h}{e^{2}}\right)^{2} \sigma_{y y}^{\mathrm{CF}}(\vec{p}, \omega=0) .
$$

This correction term gets comparable to the zeroth order term when $\left(\eta k_{\mathrm{F}}^{\mathrm{CF}} l_{\mathrm{CF}}\right)^{2} \approx 1$. Herein is $l_{\mathrm{CF}}$ the mean free path, and $k_{\mathrm{F}}^{\mathrm{CF}}$ is the Fermi wave vector of CFs. This condition is satisfied in experiment for modulation amplitudes larger than 1\%-2\%. Numerical and analytical results for the identical problem were also reported by von Oppen et al. [9]. Substituting the experimental parameters relevant for Figs. 1 and 3 in the expressions of Ref. [8] yields the dashed curves as best fits to the data. The only parameters left are the modulation magnitude $\eta$ and the resistivity $\rho_{0}^{\mathrm{CF}}$ at $B_{\text {eff }}=0$, which is expected to be higher than for an unpatterned sample in the case of etched gratings due to the damage induced by the fabrication process [14]. Note the strong damping of the oscillations predicted by theory in agreement with experiment. Only the first minimum with index $\lambda=1$ can be discerned for these state-of-the-art heterostructures.

An internal consistency check for $\eta$ is possible (under the assumption of equal $\Delta n_{s}$ at $B=0$ and $B_{\text {eff }}=0$ ) by turning our attention to the low field regime, because the damping of the electrostatic commensurability oscillations with decreasing magnetic field allows an estimate of the modulation amplitude. By lack of a better quantitative theory hitherto, a fit of the data to the exact analytical solution of the Boltzmann equation under the assumption of isotropic scattering has been employed previously for this purpose, despite a large discrepancy between the predicted linear damping [11] and the far stronger damping observed in experiment. Mirlin and co-workers, however, have recently put forward analytical expressions for a case closer to the experimentally relevant situation, when small angle scattering dominates [15]. They obtain a more satisfactory description of the low field data. Although an accurate fit to the low field data also suffers from the uncertainty in the value of the zero field resistivity after patterning, the estimates of $\eta$ are quite consistent with those extracted from fitting the high field data. The model with isotropic scattering clearly underestimates the modulation amplitude and gives values that are typically up to 3 times smaller.

The largest qualitative deviation between theory and experiment occurs close to half filling. The theory reveals no magnetic field dependence, whereas the experiment shows a positive magnetoresistance reminiscent of that observed for electrons moving in a periodic magnetic field [4]. It is believed that snake orbits, particle trajectories 
wiggling around lines of zero total effective magnetic field (inset Fig. 1), cause this positive magnetoresistance. It saturates as soon as the average effective magnetic field exceeds the amplitude of the periodic effective magnetic field component $\eta B_{1 / 2}$. This appears consistent with the experimental data. The description of the positive magnetoresistance is beyond the perturbative-in$\eta$ calculation of Ref. [8]. For the data in graph 3(c) as well as the data reported in Ref. [7], the modulation amplitude is so large that this effect persists beyond the position of the magnetic commensurability minima. As a result, the latter are suppressed, since they live from closed orbits, whereas the positive magnetoresistance relies on open runaway trajectories. For such cases, the agreement with theory, not including the effect of open snake orbits, is much poorer.

Thus far, a sinusoidal modulation has been implicitly assumed. The low field data confirms the validity of this assumption by the absence of additional commensurability features at magnetic field values given by Eq. (1) for multiples of the grating period [inset, Fig. 3(a)]. Higher order Fourier components have been intentionally suppressed in the etched gratings to prevent complications in the interpretation of the high field data [16]. This was achieved by choosing a heterostructure with a 2DES $d=220 \mathrm{~nm}$ underneath the crystal surface and by restricting the periodicities $a$ to values smaller than $2 \times d$. Holographic modulation inherently produces a sinusoidal potential when saturation is avoided.

In conclusion, we have shown that the response in $\mathrm{dc}$ transport of CFs to a periodic density modulation is dominated by the consequently induced periodic effective magnetic field. It is composed of two contributions: the long searched for oscillatory behavior due to the commensurability between the effective cyclotron radius and the lattice period of the periodic effective magnetic field and a positive magnetoresistance associated with the existence of open orbits close to $\nu=1 / 2$.

We are grateful to A. D. Mirlin, P. Wölfle, F. von Oppen and A. Stern for helpful discussions. We thank U. Waizmann and M. Riek for technical assistance. This work has been supported by the Bundesministerium für Bildung und Forschung. One of us (V. Umansky) acknowledges support from the German Israeli Foundation.

Note added in proof. - After submission of this article numerical results including the effect of open orbits were reported by Zwerschke and Gerhardts [17].
[1] B. I. Halperin, P. A. Lee, and N. Read, Phys. Rev. B 47, 7312 (1993).

[2] For example, in R. L. Willet, R. R. Ruel, K. W. West, and L. N. Pfeiffer, Phys. Rev. Lett. 71, 3846 (1993); W. Kang, H. L. Störmer, and L. N. Pfeiffer, ibid. 3850 (1993); J. H. Smet et al., Phys. Rev. Lett. 77, 2272 (1996).

[3] P. Vasilopoulos and F. M. Peeters, Superlattices Microstruct. 7, 393 (1990).

[4] P. D. Ye et al., Phys. Rev. Lett. 74, 3013 (1995).

[5] H. A. Carmona et al., Phys. Rev. Lett. 74, 3009 (1995).

[6] R. L. Willett, K. W. West, and L. N. Pfeiffer, Phys. Rev. Lett. 78, 4478 (1997).

[7] J. H. Smet, K. von Klitzing, D. Weiss, and W. Wegscheider, Phys. Rev. Lett. 80, 4538 (1998).

[8] A.D. Mirlin, P. Wölfle, Y. Levinson, and O. EntinWohlman, Phys. Rev. Lett. 81, 1070 (1998).

[9] F. von Oppen, A. Stern, and B. I. Halperin, Phys. Rev. Lett. 80, 4494 (1998).

[10] D. Weiss, K. von Klitzing, K. Ploog, and G. Weimann, Europhys. Lett. 8, 179 (1989).

[11] R. R. Gerhardts, Phys. Rev. B 53, 11064 (1996), and references therein.

[12] The same mechanism is thought to be responsible for the disparity of almost 2 orders of magnitude in the zero field resistivity and the resistivity at half filling of an unmodulated 2DES [1]. The remote positively charged donor impurities induce a static fluctuation in the particle density, which simultaneously gives rise to a spatially inhomogeneous effective magnetic field. Not the usual screened impurity scattering potential, but rather the, predominantly, large angle scattering off static fluctuations in the fictitious gauge field governs the longitudinal resistivity at $B=B_{1 / 2}$.

[13] If one would compare the influence on $\rho_{x x}$ at low fields of a periodic electrostatic potential with an amplitude $\Delta V=$ $E_{\mathrm{F}} \Delta n_{s} / \bar{n}_{s}$ with the effect on $\rho_{x x}$ of a periodic magnetic field with an amplitude $\Delta B=B_{1 / 2} \Delta n_{s} / \bar{n}_{s}$, correlated in the same way to $\Delta V$ as for $\mathrm{CFs}$, one finds using the albeit oversimplified-model of isotropic scattering [11] that the influence of the periodic magnetic field is $4 a / \lambda_{\mathrm{F}}$ larger or almost 2 orders of magnitude for the case of Fig. 1 (with $\lambda_{\mathrm{F}}$ the Fermi wavelength).

[14] It cannot simply be taken from the experiment, since a second contribution (see further in the text) not incorporated in the theory of Ref. [8] also modifies its value.

[15] A. D. Mirlin and P. Wölfle, cond-mat/9802288,

[16] A small higher order component may have substantial weight in comparison with the fundamental period near half filling due to the short mean free path of only a few times $a$.

[17] S. D. M. Zwerschke and R. R. Gerhardts, preceding Letter, Phys. Rev. Lett. 83, 2616 (1999). 